\title{
Estrategia pedagógica \\ una herramienta para el desarrollo de habilidades de la vida diaria en adultos con discapacidad intelectual
} Strategy for strengthening autonomy and independence in adults with
intellectual disabilities

(c) $\frac{1}{\mathrm{BY}}$ (): (2)

\section{Yannis Vanessa Castro Bejarano}

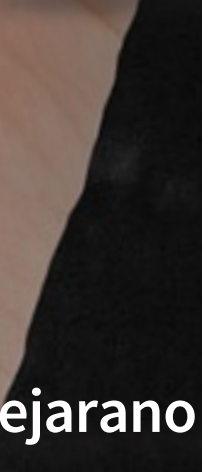




\section{HORIZONTES \\ PEDAGÓGICOS}

ID:

0123-8264.hop.21203

Title: $\quad$ Strategy for strengthening autonomy and independence in adults with intellectual disabilities

Título: Estrategia pedagógica una herramienta para el desarrollo de habilidades de la vida diaria en adultos con discapacidad intelectual

Alt Title / Título alternativo:

[en]: $\quad$ Strategy for strengthening autonomy and independence in adults with intellectual disabilities

[es]: $\quad$ Estrategia para el fortalecimiento de la autonomía e independencia en adultos con discapacidad intelectual

Author (s) / Autor (es):

Castro Bejarano

Keywords / Palabras Clave:

[en]: $\quad$ institutionalized adults; intellectual disability; independence skills; autonomy; quality of life; daily life skills

[es]: $\quad$ adultos institucionalizados; discapacidad intelectual; habilidades de independencia; autonomía; calidad de vida; habilidades de la vida diaria

Proyecto / Project:

Estrategia para el fortalecimiento de la autonomía e independencia en adultos con discapacidad intelectual

Submited: 2019-02-21

Acepted: $\quad$ 2019-09-15

\section{Resumen}

Este articulo evidencia un estudio realizado sobre el diseño y ejecución de una estrategia pedagógica como herramienta para el desarrollo de habilidades de la vida diaria de adultos con discapacidad intelectual que habitan en una institución permanentemente. Donde por medio de

la observación participante y diarios de campo, se permitió hacer un análisis pedagógico por medio de la aplicación de una batería pedagógica previamente modificada y estandarizada bajo el marco de inclusión del ministerio de educación nacional. Adicional los actores sociales que hicieron parte de la muestra de estudio, estuvo formada por 8 adultos con discapacidad intelectual que habitan permanentemente en una institución, se hizo un estudio bajo un enfoque netamente cualitativo, lo que permitió evidenciar que mediante el diseño, aplicación y evaluación de la estrategia pedagógica, se generó una transformación positiva en el desarrollo de las rutinas de la vida diaria de los actores sociales muestra de estudio, permitiendo en cada sujeto una mejor calidad de vida.

\section{Abstract}

This article evidences a study carried out on the design and execution of a pedagogical strategy as a tool for the development of daily life skills of adults with intellectual disabilities who live in an institution permanently. Where, through participant observation and field diaries, $a$ pedagogical analysis was allowed through the application of a previously modified and standardized pedagogical battery under the inclusion framework of the Ministry of Nationa Education.

In addition, the social actors who were part of the study sample, consisted of 8 adults with intellectual disabilities permanently living in an institution, a study was conducted under a purely qualitative approach, which allowed to show that through design, application and evaluation of the pedagogical strategy, a positive transformation was generated in the development of the daily life routines of the social actor's study sample, allowing in each subject a better quality of life.

\section{Citar como:}

Castro Bejarano, Y. V. (2019). Estrategia pedagógica una herramienta para el desarrollo de habilidades de la vida diaria en adultos con discapacidad intelectual. Horizontes Pedagógicos issn-l:0123-8264, 21 (2), [pgln][pgOut]. Obtenido de: https://horizontespedagogicos.ibero.edu.co/article/view/1563

Lic Yannis Vanessa Castro Bejarano, [Med]

ORCID:

$$
\text { 0000-0001-9403-3660 }
$$

\section{Source | Filiacion:}

Corporación Universitaria Iberoamericana

\section{BIO:}

Licenciada en Educación Especial de la Corporación Universitaria Iberoamericana. Diplomado Construyendo Capacidades para el Buen Vivir. Candidata a maestría en Educación con énfasis en inclusión. Joven investigadora de la Corporación Universitaria Iberoamericana

\section{City | Ciudad:}

Bogotá DC [Co]

\section{e-mail:}

ycastrob@iberoamericana.edu.co 


\section{Estrategia pedagógica una herramienta para el desarrollo de habilidades de la vida diaria en adultos con discapacidad

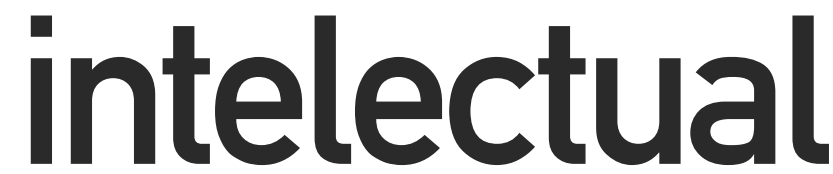

Strategy for strengthening autonomy and independence in adults with intellectual disabilities

Yannis Vanessa Castro Bejarano

\section{Introducción}

La institucionalización o habitad permanente de adultos con discapacidad intelectual en instituciones privadas o públicas, ha permitido evidenciar que son mecanismos de protección para esta población, pero que usualmente la mayoría de estas entidades se han enfocado en liderar procesos de intervención que podrían considerarse bajo modelos de rehabilitación, pues en el interior de los centros la mayoría del personal a cargo de los adultos, son profesionales del área de la salud, lo cual ha permitido inferir que las labores que se realizan estén centradas bajo inclinaciones sobreprotectoras, lo cual promueve que estos procesos estén centrados en el asistencialismo. Pues de acuerdo a lo que refiere (Palacios, 2008), el asistencialismo aparta los espacios para que las personas, en este caso los adultos con discapacidad intelectual, se puedan desempeñar de manera autónoma e independiente en las actividades de la vida cotidiana como: bañarse solo, cepillarse los dientes, elegir la ropa diariamente, vestirse solo, elegir sus alimentos, tomar decisiones, conocer y poder tener acceso a un medio de transporte público entre otras habilidades, que permiten el desarrollo personal y social, pues se ofrecen espacios reducidos para el trabajo individualizado lo cual impide el goce y desarrollo de habilidades de la vida diaria.

Este fenómeno se pudo comprobar bajo la observación participante y el análisis pedagógico en la institución donde se desarrolló la investigación, pues se pudo evidenciar que los adultos con discapacidad intelectual institucionalizados de allí, asumían rutinas masificadas y asistencialistas, esto debido a las rutinas compartidas, los horarios y dinámicas grupales impuestos por la institución, lo cual genero un tipo de asistencialismo, ya que los adultos institucionalizados no reconocen el correcto uso ni la importancia de las actividades a realizar en la vida diaria dentro y fuera de su contexto, es decir, que en el momento en que algún adulto con discapacidad tenga la oportunidad de enfrentarse a una situación dentro o fuera de la institución no sabrá cómo actuar, pues dentro de la institución siempre ha tenido un acompañamiento permanente de alguien que le suple y realiza las actividades de la vida diaria. 
Por anterior, se pudo inferir que la intervención de adultos con discapacidad intelectual, dentro de las instituciones, se desarrolla de manera pragmática, es decir que se enfocan en satisfacer las necesidades básicas, dejando de lado el componente humano y pedagógico de los adultos y a su vez anulando la edad cronológica del sujeto, siendo estos elementos un medio fundamental en el desarrollo de habilidades de vida diaria, ya que promueven un desarrollo humano digno y una mejor calidad de vida en su desenvolvimiento cotidiano, en el contexto social, personal, familiar, laboral, económico, pues de acuerdo a lo que plantea (Nussbaum, 2017) las capacidades y habilidades del ser humano son un conjunto de oportunidades que le permite a la persona actuar, decidir, elegir, promoviendo una calidad de vida idónea en la persona.

Por lo tanto, se requieren acciones que permitan darle solides al campo investigativo, por lo que se realizó una exhaustiva revisión de la literatura científica que permitió categorizar y evidenciar las contradicciones que se tienen en algunas de estas instituciones, pues aún se ven enmarcadas en modelos como la presidencia, modelo que fue instaurado por la iglesia, teniendo una mirada individual del sujeto, es decir que la persona con discapacidad era apartado de la sociedad por su condición, aspecto que aún se ve en las instituciones donde se apartar los sujetos en diferentes espacios de acuerdo a su condición, adicional lo relacionan con el modelo rehabilitador el cual fue instaurado por la medicina, modelo que entiende la discapacidad como una enfermedad, es decir que se pretende normalizar o rehabilitar al sujeto para que así pueda enmarcarse en los estándares de la sociedad. (Palacios, 2008)

\section{La sociedad decide prescindir de las personas con dis- capacidad, ya sea a través de la aplicación de políticas eugenésicas, o ya sea situándolas en el espacio destinado para lo anormales y las clases pobres, con un denominador marcado por la dependencia y el sometimiento, en el que asímismo son tratadas como objeto de caridad y sujetos de asistencia. (p.26)}

Estos modelos utilizados por algunas instituciones no comprenden la importancia del componente pedagógico mediante el cual se pueden potenciar las habilidades de la vida diaria que permitirán un desenvolvimiento en la sociedad de los adultos con discapacidad intelectual que se encuentran institucionalizados. Por lo tanto para el desarrollo de la investigación se tomó como referente el modelo social ya que de acuerdo a lo que plantea (Verdugo Alonso, 2003) "es una elaboración teórica que surgió como consecuencia de las luchas por la vida independiente y ciudadanía o derechos civiles para las personas con discapacidad"(p.238) Es entonces donde este modelo se centra en la capacidad para así generar calidad de vida, la cual va mediada por la adquisición de habilidades de la vida diaria, la autonomía e independencia, centrándose en apoyos para cada sujeto desde lo cognitivo, afectivo y social genere un desarrollo social y personal de su vida.

Apoyado a su vez en dar un paso a trabajar para romper barreras e imaginarios respecto al desenvolvimiento idóneo en la sociedad de los adultos con discapacidad intelectual, partiendo del principio humano de una vida digna, como bien lo plantea la convención sobre los derechos de las personas con discapacidad (Naciones Unidas, 2006) donde su propósito es "promover, proteger y asegurar el goce pleno y en condiciones de igualdad de todos los derechos humanos y libertades fundamentales por las personas con discapacidad, y promover el respeto de su dignidad inherente".(art.1)

Para dar un mejor soporte a lo expuesto anteriormente también se toma como eje principal frente a la conceptualización de discapacidad la postura de (Andrade, 2017) quien pone "entre comillas "dis" para poner en duda este prefijo y Capacidad con mayúscula haciendo referencia a la conversión ortográfica de los nombres propios y resaltando la capacidad de los seres humanos" (p.25), Ya que al articular las posturas expuestas anteriormente se puede afirmar que independientemente que el sujeto o el adulto tenga una discapacidad en este caso intelectual, o este en un rango de edad adulta, se le debe reconocer y brindar una intervención desde su potencial o capacidad para desarrollar habilidades de la vida diaria. Por consiguiente, también se abordó la discapacidad desde la mirada de (Vanegas Garcia \& Gil Obando, 2007) como una integración bio-psico-social, ya que se entiende "como un conjunto de elementos biológicos, sociales y culturales que conforman interrelaciones en donde todas se ven afectadas si una de ellas cambia”(p.52)

Adicional, la literatura científica consultada permitió hacer un barrido conceptual evidenciando la transformación que ha tenido la discapacidad intelectual, aunque en la actualidad aún se mantienen varios aspectos del pasado, un claro ejemplo se sustenta en lo que plantea Maldonado respecto al retardo mental o discapacidad intelectual pues refiere que no se debe tomar como un rasgo propio de la persona, sino como la expresión de la interacción de la persona con limitaciones intelectuales y adaptativas con el ambiente, haciendo énfasis en los apoyos. Citado por (Garcia Alonso, 2005). Por otro lado Luckasson refiere que la discapacidad intelectual "se caracteriza por limitaciones significativas en el funcionamiento intelectual y en la conducta adaptativa, expresada en habilidades adaptativas, conceptuales, sociales y prácticas" (Shalock, 2007, pág. 8), lo cual permite ver que este cambio se centra en la persona, resaltando la interacción entre la persona y su ambiente, esto quiere decir que se debe centrar en los apoyos que permitan una mejora del funcionamiento del individuo.

A su vez se toma la postura de (Schalock \& Verdugo, 2007) quien expresa que el ser humano es un agente de cambio entorno a la autonomía y la independencia, la cual se trabaja para un desarrollo de capacidades y habilidades donde se favorece a la persona y su productividad, generando en este caso que los adultos con discapacidad intelectual alcancen y lleven un proyecto de vida que les permita fortalecer su autoestima, sus capacidades, promoviendo una mejor calidad de vida en su entorno.

En lo que respecta a la revisión de la literatura frente a investigaciones que contemplan el fortalecimiento de autonomía e independencia para el desarrollo de habilidades de la vida diaria, se resaltan las siguientes "Discapacidad, edad adulta y vida independiente un estudio de casos", (Liesa Orús \& Vived Conte, 2010), "Potenciación de la autonomía en personas con discapacidad intelectual desde la perspectiva de los derechos humanos" (Tello Alcaide \& Sancho Frias, 2011). "Posibilidad de autonomía en adolescentes con discapacidad intelectual inhabilitante" (Daher, 2014).Manual sobre discapacidad intelectual para padres y madres de familia. Zamarron Serratos (2012). La revisión y explicación de la literatura científica aquí referida permite indicar la necesidad de realizar estudios frente a las habilidades de la vida diaria de los adultos con discapacidad intelectual, impulsando procesos de autonomía e independencia que faciliten su desarrollo personal y social, pues a pesar de que existen algunas investigaciones frente a las habilidades de la vida diaria, no se ha investigado lo suficiente, para afirmar que este campo está consolidado en su totalidad.

Por consiguiente, una de las vías fundamentales que deja la perspectiva pedagógica desde la educación especial es plantear el diseño y la implementación de una estrategia pedagógica para adultos con discapacidad intelectual como herramienta pedagógica que posibilite potenciar habilidades de autonomía e independencia para el desarrollo de habilidades de la vida, herramienta que permite generar un impacto no solo en los educadores especiales o en los adultos con 
discapacidad intelectual, sino que permite una transformación en las profesiones afines con la intervención de esta población permitiendo un trabajo experiencial, puesto que de acuerdo a lo que afirma (Fasce, 2006)

\section{Los adultos otorgan más valor a los aprendizajes que se vinculan con aquellas demandas inherentes a su quehacer de la vida diaria es decir que los adultos valoran un poco más el aprendizaje que va desde la experiencia, el que los acerca a su quehacer diario (P.69)}

Por lo anterior, se toma como categoría de análisis principal la estrategia la cual de acuerdo a la conceptualización que brinda el diccionario de la Lengua Española (Espasa, Diccionario de la lengua española, 2001) señala que la estrategia "es el arte de dirigir las operaciones militares, arte, traza para dirigir un asunto, conjunto de las reglas que aseguran una decisión óptima en cada momento" (p.1002), de igual manera, en el libro Enciclopedia de pedagogía, el mismo autor (Espasa, 2002) señala:

\section{Una estrategia de aprendizaje son reglas que permiten tomar las decisiones adecuadas en el momento oportuno en relación con el aprendizaje. Las estrategias tienen un carácter propositivo, intencional; implican, por tanto, y de forma inherente, un plan de acción, frente a las técnicas que son marcadamente mecánicas y rutinarias. Forman un conjunto de operaciones mentales: selección organización, transfer, planificación, que realiza el alumno cuando se enfrenta a su tarea de aprendizaje con el propósito de op- timizarlo. Las estrategias facilitan la adquisición, procesa- miento, transformación y recuperación de la información. Tienen un carácter intencional y están sujetas a entrena- miento. (p.1045)}

Es decir que una estrategia es una vía, un camino organizado con objetivos y acciones para materializar y lograr algo, ya que de acuerdo a la postura de Gastón Mialaret en el Diccionario de ciencias de la educación (Mialaret, 1984)., define estrategia como "la ciencia o arte de combinar y coordinar las acciones con vistas a alcanzar una finalidad. Corresponde a una planificación para lograr un resultado con proposición de objetivos a alcanzar y medios considerados para lograrlo" (p.213). Es entonces donde se considera que una estrategia pedagógica es la articulación y ejecución de los objetivos planteados donde por medio de actividades plenamente organizadas y planificadas determinará la pertinencia de esta.

\section{Metodología}

El presente estudio está enmarcado en el enfoque cualitativo, ya que según Jiménez (2000) "los métodos cualitativos parten del supuesto básico de que el mundo social está construido de significados y símbolos. De ahí la intersubjetividad sea una pieza clave de la investigación cualitativa y punto de partida para captar reflexivamente los significados sociales" (p.57) Es decir, que este enfoque es flexible y dialéctico, lo cual permitió a la investigadora, desarrollar el proceso con mayor flexibilidad, permitiendo adaptar el uso de las técnicas y procedimientos, desde el diseño, la aplicación y control de la estrategia, a través de una batería pedagógica.

El diseño seleccionado para el presente estudio es Investigación-acción participativa ya que de acuerdo a la teoría científica con- sultada se puede inferir que la finalidad de la investigación acción es (Salgado Levano, 2007) aportar información que guie la toma de decisiones para programas, procesos y reformas estructurales, que generen una transformación social. Por lo cual la autora toma como referente a Stringer citado por (Salgado Levano, 2007) quien expone que para la investigación acción hay tres fases fundamentales los cuales son: "Observar (construir un bosquejo de problemas y recolectar datos), pensar (analizar e interpretar) y actuar (resolver problemas e implementar mejoras) las cuales se dan de manera cíclica una y otra vez hasta que el problema este resuelto" (p.73)

\section{Muestra}

La muestra estuvo formada por 8 adultos con discapacidad intelectual, que están institucionalizados por más de 5 años.

Los adultos con discapacidad intelectual son atendidos bajo un enfoque asistencialista y presentan muy bajo desarrollo de las habilidades de independencia y autonomía frente al desarrollo de habilidades de la vida diaria, son bastante dependientes de los profesionales de la institución.

\section{Técnicas}

La información de esta investigación fue recolectada a través de la observación participante ya que de acuerdo a la literatura científica esta permite hacer descripciones detalladas de conductas, como de las interacciones personales y los procesos de las personas (Castro \& Rivarola, 1998), adicional la observación participante se entiende como "una estrategia del investigador para mirar, acercarse e interpretar la realidad social investigada y es, simultáneamente, una táctica de campo la cual se comprende desde dos dimensiones: la epistemológica y la técnica" (Licona Valencia \& Garcia Sotelo, pág. 254). Es decir que el investigador debe crear una relación social cercana con los sujetos o individuos que hacen parte de la investigación.

la batería pedagógica, batería que fue diseñada y adaptada desde el documento de Orientaciones técnicas administrativas y pedagógicas para la atención educativa de personas con discapacidad en el marco de la educación Inclusiva (Ministerio de Educación Nacional, 2017).

\section{Procedimientos}

A continuación, se describen las fases de la investigación:

La primera fase de reflexión, para la que como instrumentos se utilizó la batería pedagógica que estuvo compuesta por descripciones iniciales, análisis críticos y analíticos lo cual permitió realizar un contraste con los resultados previos al desarrollo del proyecto.

La segunda fase de construcción, fue desarrollada desde el análisis inicial de los participantes del proyecto y su entorno replanteando de esta manera como educadora planes de acción realizados de manera práctica y secuencial en cada uno de los encuentros fortaleciendo una relación directa con el problema de investigación. Para este proyecto se determinó como uno de los criterios de mayor complejidad para el desarrollo de actividades de la vida diaria la institucionalización de los participantes, es por lo que mediante la observación participante se buscó la inclusión de la investigación en el proceso y en el desarrollo del proyecto de manera activa y concreta, se rescató la familiarización 
presencial de las situaciones expuestas teóricamente, generando experiencias vivenciales en los participantes.

Tercera fase recolección de evidencias, análisis, evaluación y entrega de resultados se contó con la batería pedagógica que tuvo la descripción detallada de cada una de las aplicaciones con cada uno de los resultados y criterios de evaluación utilizados durante el proceso, luego de ejecutar la estrategia pedagógica.

El grupo parte de la muestra compuesto por 8 adultos con discapacidad intelectual institucionalizados, participaron durante año y medio, con una intensidad horaria de 5 horas semanales bajo un esquema de enseñanza- aprendizaje- experiencia, donde se abordaron actividades de la vida cotidiana. Cabe resaltar que las actividades expuestas para cada encuentro fueron previamente planeadas y estructuradas en la estrategia pedagógica elaborada, y su aplicación se hizo bajo la observación, para ver la efectividad o no de la estrategia y si era necesario reestructurar la misma o la manera de su aplicación. Durante el desarrollo de las actividades y acciones, se creaban situaciones nuevas que eran atendidas en el mismo contexto, ya que, por ser un diseño cualitativo, el investigador podía hacer esto, y además eran acciones de tipo pedagógica, que buscaban mayor efectividad en el proceso de desarrollo de los participantes.

De igual manera junto a la observación participante se aplicó la batería pedagógica, batería que fue diseñada y adaptada desde el documento de Orientaciones técnicas administrativas y pedagógicas para la atención educativa de personas con discapacidad en el marco de la educación Inclusiva. Instrumento que permitió el estudio sobre las habilidades en torno a la independencia y la autonomía en la vida diaria, antes de iniciar la aplicación de la estrategia para ver el nivel de desarrollo de estas habilidades, y posteriormente, luego de aplicar la estrategia, se volvió a aplicar la batería para a partir de los resultados, ver la efectividad de la misma en el desarrollo de las habilidades de autonomía e independencia.

La estrategia pedagógica, diseñada y aplicada constó con sus objetivos estratégicos, su dimensión estratégica y su estructura, en la cual se concibió el momento del diagnóstico, para caracterizar el estado actual del desarrollo de las habilidades de los adultos objeto de estudio, en torno a las habilidades de independencia y autonomía. A partir de esa caracterización se procedió a hacer el sistema de actividades de la estrategia, dentro de su etapa de planeación, estas actividades se hicieron a partir de los resultados del diagnóstico. Seguidamente se concibió la etapa de la aplicación de la estrategia y finalmente su control, mediante la aplicación de la batería pedagógica y la observación para determinar la eficacia de la misma.

\section{Análisis de resultados}

A continuación se realiza el análisis de los datos que se obtuvieron de la observación participante en cada uno de los momentos que se hizo presencia en la institución; esta observación participante en la aplicación de la batería pedagógica inicial, permitió corroborar en la práctica que el modelo que se estaba llevando a cabo dentro de la institución, era un modelo asistencialista, lo cual generaba que los adultos con discapacidad intelectual que allí están institucionalizados, mostraran conductas de dependencia a terceros, lo cual generaba que los adultos tuvieran una autonomía muy limitada.

Esto se observó en los diferentes roles que desempeñaban dentro de la casa, permitiendo evidenciar que la mayoría de los adultos necesitaban ayuda para realizar actividades básicas de la vida diaria como: bañarse los dientes, bañarse, secarse, elegir su ropa, vestirse, elegir sus alimentos, tomar decisiones, arreglar sus cuartos, desplazarse por sí solos dentro de la institución etc, adicional que todas estas actividades las realizaban de manera masificada, lo cual limitaba aún más su independencia y autonomía dentro de la institución.

Esto permitió en la investigación concebir desde la educación especial y la observación participante, la necesidad de transformar esa situación, mediante una intervención pedagógica, ya que esta problemática estaba afectando directamente la autonomía, independencia y la calidad de vida de los adultos allí institucionalizados. La vía que se tomó para la transformación de esta intervención fue el diseño y ejecución de una estrategia pedagógica la cual se diseñó y se aplicó. Posteriormente se procedió analizar los datos obtenidos, sin embargo, no se utilizó ningún programa matemático, ni de control de variables desde una perspectiva numérica, ya que el análisis fue netamente descriptivo, lo cual permitió registrar de manera cualitativa el contraste de los resultados

\section{Resultados obtenidos a partir de la puesta en práctica de la batería pedagógica.}

Los datos obtenidos mediante la observación y la aplicación de la batería pedagógica, batería diseñada y adaptada desde el documento Orientaciones técnicas administrativas y pedagógicas para la atención de estudiantes con discapacidad en el marco de la educación inclusiva (Ministerio de Educación Nacional, 2017) fueron procesados y los resultados obtenidos fueron objeto de análisis cualitativo. Estos datos aparecen en las tablas que a continuación se presentan.

Tabla 1 Se puede observar la rúbrica de evaluación donde se relacionan los parámetros que se tendrán en cuenta para la aplicación de la batería pedagógica

\begin{tabular}{|c|c|c|c|}
\hline Calificador & $\begin{array}{c}\text { Escala } \\
\text { cualitativa }\end{array}$ & Apoyo & Observaciones \\
\hline No hay dificultad & Superior & No requiere apoyo & $\begin{array}{l}\text { Cuando el sujeto domina plenamente la habilidad sin apoyo } \\
\text { del docente. }\end{array}$ \\
\hline Dificultad ligera & Alto & Intermitente & $\begin{array}{l}\text { Cuando el sujeto es capaz de realizar la actividad pero requiere } \\
\text { de un acompañamiento ocasional o escaso. }\end{array}$ \\
\hline Dificultad moderada & Bajo & Limitado & $\begin{array}{l}\text { Cuando el sujeto presenta avances, pero requiere de un apoyo } \\
\text { por un tiempo determinado para la ejecución autónoma e } \\
\text { independiente de la actividad. }\end{array}$ \\
\hline Dificultad grave & Escaso & Extenso & $\begin{array}{l}\text { Cuando el sujeto aún presenta dependencia para la realización } \\
\text { de ciertas actividades por lo cual requiere un apoyo a largo } \\
\text { plazo. }\end{array}$ \\
\hline
\end{tabular}


Tabla 2 Tabla comparativa de la aplicación de la batería pedagógica antes y después de la aplicación de la estrategia pedagógica en el sujeto 1.

\begin{tabular}{lcc}
$\begin{array}{l}\text { ANÁLISIS CUALITATIVO DE LOS RESULTADOS DURANTE EL PROCESO DE EJECUCIÓN DE LA } \\
\text { ESTRATEGIA PEDAGÓGICAS. }\end{array}$ & $\begin{array}{l}\text { Caracterización inicial } \\
\text { SUJETO } 1\end{array}$ & $\begin{array}{c}\text { Expresión de la influencia de } \\
\text { desarrollo de la estrategia }\end{array}$ \\
\hline Habilidades Cognitivas & Dificultad Moderada & No Hay Dificultad \\
\hline Habilidades Comunicativas & Dificultad Grave & No Hay Dificultad \\
\hline Conducta Adaptativas & Dificultad Ligera \\
\hline $\begin{array}{l}\text { Personalidad y habilidades sociales } \\
\text { Habilidades Motoras }\end{array}$ & Dificultad Grave & Dificultad Ligera \\
Sexualidad & Dificultad Moderada & Dificultad Moderada
\end{tabular}

Fuente. Elaborada por la autora, basada en los resultados obtenidos de la aplicación de las baterías pedagógicas, pruebas pre-test y pos-test.

Donde se puede observar como el sujeto manifestó un desarrollo y un fortalecimiento positivo en las habilidades adaptativas y una mejora considerable, en cada una de las habilidades evaluadas después de aplicada la estrategia pedagógica, generando un avance evidente en las habilidades cognitivas y comunicativas, llevando al sujeto a tener mayor independencia y autonomía.

Tabla 3 Tabla comparativa de la aplicación de la batería pedagógica antes y después de la aplicación de la estrategia pedagógica en sujeto 2

\begin{tabular}{lcc} 
SUJETO 2 & Caracterización inicial & $\begin{array}{c}\text { Expresión de la influencia de } \\
\text { desarrollo de la estrategia }\end{array}$ \\
Habilidades Cognitivas & Dificultad Moderada & Dificultad Ligera \\
\hline Habilidades Comunicativas & Dificultad Grave & Dificultad Ligera \\
Conducta Adaptativas & Dificultad Completa & Dificultad Ligera \\
\hline Personalidad y habilidades sociales & Dificultad Completa & Dificultad Ligera \\
Habilidades Motoras & Dificultad Grave & Dificultad Moderada \\
Sexualidad & Dificultad Moderada & Dificultad Ligera
\end{tabular}

Fuente. Elaborada por la autora, basada en los resultados obtenidos de la aplicación de las baterías pedagógicas, pruebas pre-test y pos-test.

Se puede observar el contraste que se tiene el antes y el después de la aplicación de la estrategia, la cual deja evidenciar el desarrollo positivo que se tuvo no solo en las habilidades adaptativas, sino como al fortaleces dichas habilidades se genera una mejora en las demás habilidades.

Tabla 4 Tabla comparativa de la aplicación de la batería pedagógica antes y después de la aplicación de la estrategia pedagógica en sujeto 3.

\begin{tabular}{lcc} 
SUJETO 3 & Caracterización inicial & $\begin{array}{c}\text { Expresión de la influencia de } \\
\text { desarrollo de la estrategia }\end{array}$ \\
Habilidades Cognitivas & Dificultad Grave & Dificultad Ligera \\
\hline Habilidades Comunicativas & Dificultad Grave & Dificultad Ligera \\
\hline Conducta Adaptativas & Dificultad Completa & Dificultad Ligera \\
\hline Personalidad y habilidades sociales & Dificultad Moderada & No Hay Dificultad \\
Habilidades Motoras & Dificultad Moderada & No Hay Dificultad \\
Sexualidad & Dificultad Moderada & Dificultad Ligera
\end{tabular}

Fuente. Elaborada por la autora, basada en los resultados obtenidos de la aplicación de las baterías pedagógicas, pruebas pre-test y pos-test.

Se puede observar como el sujeto tres muestra un gran contraste en la caracterización inicial comparándolo con la expresión de la influencia de desarrollo de la estrategia, manifestando un desarrollo bastante positivo en cada una de las habilidades trabajadas.

Tabla 5 Tabla comparativa de la aplicación de la batería pedagógica antes y después de la aplicación de la estrategia pedagógica en sujeto 4

\begin{tabular}{llc} 
SUJETO 4 & Caracterización inicial & $\begin{array}{c}\text { Expresión de la influencia de } \\
\text { desarrollo de la estrategia }\end{array}$ \\
Habilidades Cognitivas & Dificultad Moderada & Dificultad Ligera \\
\hline Habilidades Comunicativas & Dificultad Moderada & Dificultad Ligera \\
Conducta Adaptativas & Dificultad Completa & Dificultad Moderada \\
\hline Personalidad y habilidades sociales & Dificultad Moderada & Dificultad Ligera \\
\hline Habilidades Motoras & Dificultad Moderada & Dificultad Ligera \\
Sexualidad & Dificultad Moderada & Dificultad Ligera
\end{tabular}


Se observa un gran resultado en el contraste que se tiene después de la aplicación de la estrategia pedagógica en este sujeto.

Tabla 6 Tabla comparativa de la aplicación de la batería pedagógica antes y después de la aplicación de la estrategia pedagógica en sujeto 5 .

\begin{tabular}{lcc} 
SUJETO 5 & Caracterización inicial & $\begin{array}{c}\text { Expresión de la influencia de } \\
\text { desarrollo de la estrategia }\end{array}$ \\
\hline Habilidades Cognitivas & Dificultad Grave & Dificultad Ligera \\
\hline Habilidades Comunicativas & Dificultad Grave & Dificultad Ligera \\
\hline Conducta Adaptativas & Dificultad Completa & Dificultad Ligera \\
\hline Personalidad y habilidades sociales & Dificultad Moderada & Dificultad Ligera \\
Habilidades Motoras & Dificultad Moderada & Dificultad Ligera \\
Sexualidad & Dificultad Moderada & Dificultad Ligera
\end{tabular}

Fuente. Elaborada por la autora, basada en los resultados obtenidos de la aplicación de las baterías pedagógicas, pruebas pre-test y pos-test

Se observa la eficacia de los resultados obtenidos en cada una de las habilidades después de aplicada la estrategia, resaltando las habilidades adaptativas en la cual presento un gran fortalecimiento en habilidades inherentes a la autonomía e independencia.

Tabla 7 Tabla comparativa de la aplicación de la batería pedagógica antes y después de la aplicación de la estrategia pedagógica en sujeto 6

\begin{tabular}{|c|c|c|}
\hline SUJETO 6 & Caracterización inicial & $\begin{array}{l}\text { Expresión de la influencia de } \\
\text { desarrollo de la estrategia }\end{array}$ \\
\hline Habilidades Cognitivas & Dificultad Moderada & No Hay Dificultad \\
\hline Habilidades Comunicativas & Dificultad Moderada & Dificultad Ligera \\
\hline Conducta Adaptativas & Dificultad Completa & Dificultad Ligera \\
\hline Personalidad y habilidades sociales & Dificultad Moderada & Dificultad Ligera \\
\hline Habilidades Motoras & Dificultad Moderada & No Hay Dificultad \\
\hline Sexualidad & Dificultad Moderada & Dificultad Ligera \\
\hline
\end{tabular}

Se observa un desarrollo favorable en cada una de las variables trabajadas antes y después de la aplicación de la estrategia.

Tabla 8 Tabla comparativa de la aplicación de la batería pedagógica antes y después de la aplicación de la estrategia pedagógica en sujeto 7

$\begin{array}{lcc}\text { SUJETO } 7 & \text { Caracterización inicial } & \begin{array}{c}\text { Expresión de la influencia de } \\ \text { desarrollo de la estrategia }\end{array} \\ \text { Habilidades Cognitivas } & \text { Dificultad Grave } & \text { Dificultad Moderada } \\ \text { Habilidades Comunicativas } & \text { Dificultad Grave } & \text { Dificultad Ligera } \\ \text { Conducta Adaptativas } & \text { Dificultad Completa } & \text { Dificultad Moderada } \\ \text { Personalidad y habilidades sociales } & \text { Dificultad Grave } & \text { Dificultad Ligera } \\ \text { Habilidades Motoras } & \text { Dificultad Moderada } & \text { Dificultad Ligera } \\ \text { Sexualidad } & \text { Dificultad Grave } & \text { Dificultad Ligera }\end{array}$

Fuente. Elaborada por la autora, basada en los resultados obtenidos de la aplicación de las baterías pedagógicas, pruebas pre-test y pos-test

Se observa que el sujeto 7 a diferencia de los demás participantes tuvo un avance menor en cada una de las habilidades, su proceso fue un poco inferior ya que tan solo logro generar un pequeño cambio, sin embargo, cabe resaltar que fue bastante significativo ver avances en cada una de las experiencias desarrolladas con este sujeto ya que lograr un poco de independencia y autonomía le permitió empoderarse en algunos aspectos de su vida cotidiana.

Tabla 9 Tabla comparativa de la aplicación de la batería pedagógica antes y después de la aplicación de la estrategia pedagógica en sujeto 8

\begin{tabular}{lcc} 
SUJETO 8 & Caracterización inicial & $\begin{array}{c}\text { Expresión de la influencia de } \\
\text { desarrollo de la estrategia }\end{array}$ \\
\hline Habilidades Cognitivas & Dificultad Moderada & No Hay Dificultad \\
\hline Habilidades Comunicativas & Dificultad Moderada & Dificultad Ligera \\
\hline Conducta Adaptativas & Dificultad Grave & No Hay Dificultad \\
\hline Personalidad y habilidades sociales & Dificultad Moderada & Dificultad Ligera \\
\hline Habilidades Motoras & Dificultad Moderada & No Hay Dificultad \\
Sexualidad & Dificultad Moderada & Dificultad Ligera
\end{tabular}


Se observa el avance tan significativo que presento este sujeto con la aplicación de la estrategia, permitiendo evidenciar un desarrollo positivo en la mayoría de las habilidades trabajadas, permitiendo afirmar que fue el sujeto 8 quien mayor avance presento.

Los resultados del estudio, en un proceso inicial de caracterización, revelan el contraste que se obtuvo en la aplicación de la batería pedagógica, permitiendo evidenciar el enfoque asistencialista que se impartía en la institución por parte de los profesionales, el cual limita, que los sujetos potencien las habilidades inherentes a la autonomía e independencia, sin embargo con esta investigación se logró ver la necesidad inmediata, de generar una transformación, para así crear espacios nuevos en los diferentes contextos de la institución, que permitiera a los adultos que allí habitan, se empoderaran en el día a día, en la realización de las actividades de la vida cotidiana.

Estas características obtenidas por medio de la observación permitieron reafirmar la importancia y la necesidad fundamental, por generar espacios para potenciar y fortalecer la autonomía y la independencia en adultos con discapacidad intelectual institucionalizados, generando en ellos una mejor calidad de vida, posibilitando en cada sujeto la toma de decisiones propias, hacia estas habilidades.

Adicional en este análisis cualitativo, se pudo observar que los resultados fueron muy buenos con relación a la estrategia diseñada, pues mediante su aplicación, y el control de su efectividad, a partir de la observación participante y de la batería pedagógica permitieron evidenciar y determinar la eficacia de la herramienta, y aunque uno en uno de los sujetos no se evidencio un avance tan significativo como en los demás participantes, cabe resaltar que para la educación especial ese cambio tan mínimo, es bastante significativo ya que permitió hacer mejoras en la estrategia, y permitió establecer que con mayor constancia en la aplicación de la prueba se pueden obtener mejores resultados.

\section{Discusión y conclusiones}

Dados los resultados se comprobó que la estrategia pedagógica diseñada y aplicada, fue eficiente y eficaz en el cumplimiento de los objetivos propuestos, lo cual constituye una experiencia positiva en el orden pedagógico para los adultos con discapacidad Intelectual Institucionalizados, permitiéndoles potenciar y fortalecer las habilidades de independencia y autonomía en el desarrollo de habilidades de la vida diaria, lo que eleva la calidad de vida de esta población.

Posteriormente se puede contrastar claramente la eficacia de esta investigación con las expuestas en el marco de referencia, "Discapacidad, edad adulta y vida independiente un estudio de casos", (Liesa Orús \& Vived Conte, 2010), "Posibilidad de autonomía en adolescentes con discapacidad intelectual inhabilitante” (Daher, 2014), "Potenciación de la autonomía en personas con discapacidad intelectual desde la perspectiva de los derechos humanos" (Tello Alcaide \& Sancho Frias, 2011), las cuales desde las diferentes variables y perspectivas coinciden y apuntan en que los centros de apoyo, las familias, los profesionales a cargo de la población con discapacidad intelectual adulta, se caracterizan por su conducta asistencialista y sobreprotectora, por lo cual es necesario crear herramientas de apoyo que permitan que los sujetos con discapacidad intelectual puedan asumir rutinas diarias de manera autónoma e independiente, que puedan empoderarse y tener un estilo de vida que les genere mejor calidad de vida, que pueda tomar decisiones acerca de sus actividades cotidianas, adicional en las cuatro investigaciones se hace un fuerte reconocimiento a los derechos fundamentales de las personas con discapacidad, basados en la convención de derechos, reconociendo al sujeto para que tenga una vida digna, empoderándolo jurídicamente, socialmente, enfocándose en la importancia de la autonomía e independencia en cada sujeto, permitiendo generar cambios, no solo en las personas con discapacidad sino también en las personas que intervienen en su día a día.

Por lo anterior se debe entender que la atención o la asistencia que se brinda por parte de los profesionales del área de la salud para los adultos con discapacidad intelectual que se encuentran institucionalizados es necesaria en las instituciones, pero muchas veces esta tendencia ofrece espacios limitados para el desarrollo de las habilidades de la autonomía e independencia, es entonces que se puede resaltar y destacar que cuando se hace un trabajo pedagógico sólido y científico, hacia este tipo de población, y no nos limitamos a la atención médica y terapéutica, los resultados son superiores en una atención integral a estos adultos, permitiendo una transformación en el enfoque asistencial, generando un trabajo interdisciplinar para así elevar la calidad de vida respecto a las habilidades inherentes a la autonomía e independencia.

Por ultimo esta investigación permitió abrir nuevos horizontes de investigación frente a los adultos con discapacidad intelectual, pues este estudio demostró la importancia de vincular no solo a la población (actores sociales de la investigación) sino de unir o asociar a profesionales de áreas inmersas a la atención de esta población, a vincular las familias, y de la importancia del trabajo interdisciplinar para el proceso de desarrollo de habilidades de la vida diaria en adultos con discapacidad intelectual.

\section{Referencias}

Andrade, F. (2017). Orientaciones conceptuales y metodologicas para la atención educativa en clave de "dis"capacidad. Bogota: Corporación universitaria Minuto de Dios-UNIMINUTO.

Castro, V., \& Rivarola, M. (1998). Manual de capacitación para talleres de trabajo sobre el uso de la Metodologia de la investigación cualitativa.

Daher, J. (Octubre de 2014). Posibiloidad de autonomia en adolecentes con discapacidad intelectual inhabilitante. Recuperado el 4 de febrero de 2019, de imgbiblio.vaneduc.edu. ar/fultext/files/TC116712.pdf

Espasa. (2001). Diccionario de la lengua española (vigésima segunda edición ed.). Madrid.

Espasa. (2002). Enciclopedia de pedagogia (Vol. 5). España: Royce Editores.

Fasce H. , E. (2006). ANDRAGOGíA. Revi. educación, Ciencias, Salud., III(2), 69-70. Recuperado el 20 de Julio de 2019, de http://www2. udec.cl/ofem/recs/anteriores/vol322006/esq32.pdf

Garcia Alonso, I. (2005). Concepto actual de la discapacidad intelectual. Psychosocial intervention, 14(3), 255-276.

Licona Valencia, E., \& Garcia Sotelo, D. (s.f.). La observación participante en la investigación social. Puebla.

Liesa Orús, M., \& Vived Conte, E. (2010). Discapacidad, edad adulta y vida independiente. Un estudio de casos. Educación y diversidad, 4(1), 101-124.

Mialaret. (1984). Diccionario de ciencias de la educación . Barcelona: Oikos-tau. 
Ministerio de Educación Nacional. (Febrero de 2017). Orientaciones técnicas administrativas y pedagógicas para la atención de estudiantes con discapacidad en el marco de la educación inclusiva. Recuperado el 15 de 09 de 2018, de http://www. mineducacion.gov.co/1759/articles-360293_foto_portada.pdf

Naciones Unidas. (13 de Diciembre de 2006). Convención sobre los derechos de las personas con discapacidad. Convención sobre los derechos de las personas con discapacidad. Nueva York.

Nussbaum, M. (2017). Crear capacidades (Tercera ed.). Barcelona: Planeta Colombia S.A.

Palacios, A. (2008). El modelo social de discapacidad: orígenes, caracterizaión y plasmación en la convención Internacional sobre los Derechos de las Personas con Discapacidad. (S. Cinca, Ed.) Madrid: Cinca.

Salgado Levano, A. (2007). Investigación culitativa; Diseños, Evaluación del rigor metodologico y retos. Liberavit, 13(13), 71-78. Recuperado el 11 de Julio de 2019, de http://www.scielo. org.pe/scielo.php?pid=S1729-48272007000100009\&script=sci arttext\&tlng=en

Schalock, R. I., \& Verdugo, M. A. (2007). El concepto de Calidad de vida en los servicios y apoyos para personas con discapacidad intelectual. Revista Española sobre Discapacidad Intelectual, 38(4), 21-36.
Shalock, R. (2007). El nuevo concepto de retraso mental: comprendiendo el cambio al termino discapacidad intelectual. Riberdis, 38(4)(224), 5-20.

Tello Alcaide, R., \& Sancho Frias, I. (28 de Junio de 2011). Potenciación de la autonomia en personas con discapacidad intelectual desde la perspectiva de los derechos humanos. Obtenido de www3.uah.es/congresoreps2013/paneles/panel4/sesión3/ isancho@urg.es/TCPONENCIAPANEL4ENVIDADA.pdf

Vanegas Garcia, J. H., \& Gil Obando, L. M. (2007). La discapacidad, una mirada desde la teoria de sistemas y el modelo biopsicosocial. Hacia la promoción de la salud, 12, 51-61.

Verdugo Alonso, M. (2003). La concepción de la discapacidad en los modelos sociales. Dialnet, 235-247.

Zamarron Serratos, Y. (2012). Manual sobre discapacidad intelectual para padres y madres de familia. Bogotá: Universidad Pedagogica Nacional. Recuperado el 09 de 06 de 2019, de http://200.23.113.51/pdf/29084.pdf 